\title{
Acknowledgement of Ad Hoc Reviewers
}

In addition to the editorial board, the following people reviewed manuscripts for Psychological Methods during the period in which volume 26 was composed. Their help is gratefully acknowledged.

\begin{tabular}{|c|c|}
\hline \multirow{2}{*}{\multicolumn{2}{|c|}{$\begin{array}{l}\text { Andre Achim } \\
\text { Samuel Aeschbach* }\end{array}$}} \\
\hline & \\
\hline Casper Albers & Edgar Erdfelder \\
\hline Sebastian Castro Alvarez* & Ashkan Erterfaie \\
\hline \multicolumn{2}{|l|}{ Royce Anders } \\
\hline Corson Areshenkoff & Leandre Fabrigar \\
\hline Frederik Aust & Carl Francis Falk \\
\hline Razia Azen & $\begin{array}{l}\text { Tom Faulkenberry } \\
\text { Yi Feng }\end{array}$ \\
\hline Donald Bacon & Emilio Ferrer \\
\hline Thom Baguley & W. Holmes Finch \\
\hline Roger Bakeman & Jessica Flake \\
\hline Zsuzsa Bakk & David B. Flora \\
\hline Dale J. Barr & Miriam K. Forbes \\
\hline Anwesha Bhattacharyya & Jean-Paul Fox \\
\hline Francis Bilson Darku & Greg Francis \\
\hline Simon Blanchard & Michael Friendly \\
\hline Ulf Bockenholt & Michael Frisby \\
\hline \multicolumn{2}{|l|}{ Robert J. Boik } \\
\hline Steven Boker & Christian Geiser \\
\hline Niall Bolger & Cees A. W. Glas \\
\hline Wes Bonifay & Hudson Golino \\
\hline Juan Botella & Joseph Edward Gonzales \\
\hline Andreas M. Brandmaier & Oscar Gonzalez \\
\hline Holger Brandt & Fei Gu \\
\hline Michael T. Brannick & Heather J. Gunn \\
\hline \multicolumn{2}{|l|}{ Timothy R. Brick } \\
\hline Laura Bringmann & Adam R. Hafdahl \\
\hline Michael J. Brusco & Fumiaki Hamagami \\
\hline & Andrew Hardin \\
\hline Math Candel & Tom Hardwicke \\
\hline Joseph Cappelleri & Jonas M. B. Haslbeck \\
\hline Daniel R. Cavagnaro & Fred Hasselman \\
\hline Eva Ceulemans & Leslie A. Hayduk \\
\hline Kuan-Hua Chen & Daniel W. Heck \\
\hline Lihan Chen* & Erin Hennes \\
\hline Po-Yi Chen & Teague Henry \\
\hline Ying (Alison) Cheng & Arianne Herrera-Bennett \\
\hline Donna L. Coffman & Joseph Hilgard \\
\hline José Cortina & Herbert Hoijtink \\
\hline Denis Cousineau & Maxwell Hong* \\
\hline Catherine Crespi & Michael D. Hunter \\
\hline Robert A. Cribbie & Heungsun Hwang \\
\hline \multicolumn{2}{|l|}{ Steven Andrew Culpepper } \\
\hline & Suzanne Jak \\
\hline Fabian Dablander & Minjeong Jeon \\
\hline Jonas Dalege & Booil Jo \\
\hline Jason Dana & Valen Johnson \\
\hline Mark L. Davison & Raquael Joiner \\
\hline Clintin Davis-Stober & Raquael Joiner \\
\hline Paul De Boeck & Payton Jeffrey Jones \\
\hline \multicolumn{2}{|l|}{ Mark de Rooij } \\
\hline Lawrence T. DeCarlo & Jeffrey S. Kane \\
\hline Jamie DeCoster & Rogier Kievit \\
\hline Christopher Desjardins & Eunsook Kim \\
\hline Zoltan Dienes & Minjung Kim \\
\hline Cody Ding & Se-Kang Kim \\
\hline Peter Dixon & Rex B. Kline \\
\hline Philipp Doebler & Stanislav Kolenikov \\
\hline Michael Dougherty & Alena Kostyk \\
\hline \multicolumn{2}{|l|}{ Jeffrey A. Douglas } \\
\hline Fritz Drasgow & Mark Lai \\
\hline
\end{tabular}

Daniel Lakens
Charles E. Lance
Roger T. A. J. Leenders
Cheri Levinson
Roy Levy
Cheng-Hsien Li
Yuelin Li
Cary Lin*
Qimin Liu
Siwei Liu
Xiao Liu
Yang Liu
Yu Liu
Jordan Loeffelman
Rosaria Lombardo
Oliver Lüdtke
Wei-Ming Luh

Yue Ma

Brooke Magnus George A. Marcoulides

Katerina M. Marcoulides

Maartin Marsman

Elena Martynova*

Rui Mata

Scott E. Maxwell

Alberto Maydeu-Olivares

Cameron McIntosh

Blakeley McShane

Catherine Mintz*

Milica Miocevic

Mariola Moeyaert

Giusi Moffa

Dylan Molenaar

Morten Moshagen

Irini Moustaki

Joris Mulder

Bengt Muthen

Trang Quynh Nguyen

Christopher Nye

Jake Olivier

Fred Oswald

Timothy J. Ozechowski

Daniel J. Ozer

Ozlem Ozkok

Soojin Park

Alexandra Paxton

Andreas Pfaffel

Steffi Pohl

Kristopher J. Preacher

MeiHua Qian

Xu Qin

Rafael Roberto Ramirez

Philippe Rast

Michel Regenwetter

Fabiola Reiber

Steven Reise
William Revelle

Jason Rights

Alexander Robitzsch

Julia Rohrer

Zachary Roman*

Jeffrey N. Rouder

John Ruscio

Oisín Ryan

Elizabeth Ann Sanders

Florian Scharf

Karin Schermelleh-Engel

Jonathan D. Schweig

Michael A. Seaman

Sarfaraz Serang

Ronald C. Serlin

Can Shao

Dexin Shi

Gwowen Shieh

Richard Shiffrin

Stephen Short

Cynthia Siew

Sandip Sinharay

Hairong Song

Vincent Staggs

Stephen Stark

Joel S. Steele

Lynne Steinberg

Rolf Steyer

Jenn-Yun Tein

Jonathan Templin

Jorge N. Tendeiro

Dave Thissen

Felix J. Thoemmes

Marieke E. Timmerman

David Trafimow

Stephen Tueller

Francis Tuerlinckx

Rolf Ulrich

Satoshi Usami

Matthew Valente

Rens van de Schoot

Wim Van den Noortgate

Sara Van Erp

Don van Ravenzwaaij

Joachim Vandekerckhove

Shravan Vasishth

Juan Diego Vera*

Wolfgang Viechtbauer

Marieke Visser

Manuel C. Voelkle

John R. Vokey

Alexander von Eye

Timo Georg von Oertzen

Eric-Jan Wagenmakers

Lourens Waldorp

Niels Waller

Chun Wang 


$\begin{array}{llll}\text { Y. Andre Wang } & \text { Carla Willig } & \text { Wei Wu } & \text { Peida Zhan } \\ \text { Isaac Joel Washburn } & \text { Michael Willoughby } & \text { Dirk Wulff } & \text { Jinming Zhang } \\ \text { Ashley Watts } & \text { Tim Wilson } & \text { Anna Wysocki* } & \text { Qian Zhang } \\ \text { Zhonglin WEN } & \text { John Wixted } & \text { Mike Zyphur } \\ \text { Keith Widaman } & \text { Phil K. Wood } & \text { Ji Seung Yang } & \\ \text { Wolfgang Wiedermann } & \text { Aidan Wright } & \text { Manshu Yang } & \\ \text { Kenneth Tyler Wilcox } & \text { Hao Wu } & \text { Ai Ye } & \end{array}$

*Denotes a reviewer who co-reviewed under the supervision of a primary reviewer. 